\title{
An outbreak of Influenza A (H1N1) 2009 at Mae Fah Luang University, Chiang Rai Province, northern Thailand
}

Tawatchai Apidechkul

From $16^{\text {th }}$ International Symposium on HIV and Emerging Infectious Diseases

Marseille, France. 24-26 March 2010

\section{Background}

Since the first outbreak of a novel influenza A (H1N1) was reported in Thailand in late April 2009, new cases have gradually increased nationwide. On 2 July 2009, Mae Fah Luang University officials were notified of many suspected cases among students who sought care at the university hospital. Severe cases were referred to a district hospital and a regional hospital. On 3 July 2009, 3 positive H1N1 PCR results were reported and immediate actions were taken to investigate and control this outbreak.

\section{Methods}

At the early morning of July 3,2009 , the epidemiologist was called for the rapid increasing of the number of patients who attended at MeaFahLuangUniversityHospital, with the signs and symptoms related to the respiratory tract infection. This might been the first epidemic of the Influenza A (H1N1) virus which looks like an outbreak on the large of infected people at the same area and the same time. The investigator went to the field and conducted disease investigation and control.

On 4 July 2009, we began to investigate the outbreak by closely monitoring patients and seeking suspected cases on the University campus where 1,115 students occupied in 32 dormitories. We developed questionnaires based on MOPH ones to collect demographical and epidemiological data. Following control measures were implemented. Patients were treated with proper care (intensive care/isolated rooms). Tamiflu and influenza vaccine were stockpiled. Face masks were distributed. Housekeepers and students were provided with AVANTA $^{\circ}$ to disinfect areas of disease contamination in

Correspondence: tk2516ms@hotmail.com

Mae Fah Luang University, Chiang Rai Province, Thailand buildings and dormitories. Knowledge on influenza and personal hygiene were emphasized. Large social gatherings were canceled. Necessary activities were conducted in well-ventilated areas. Air conditioners were not operated. Patients and suspected cases were asked to wear face masks at all times and rest at dormitories; they were closely monitored after returning to classes.

\section{Results}

During 2-7 July 2009, 165 patients (16, 33, 36, 54, 24 and 2 cases on consecutive days) were reported with signs and symptoms related to a novel influenza A (H1N1). Major signs included fever $(70.3 \%)$, cough (83.9\%), muscle pain (28.9\%) and diarrhea (4.2\%). Mean age was 19.6 years (range $16-46$ years); $68.5 \%$ were females; $58.1 \%$ and $22.9 \%$ were the $1^{\text {st }}$ and $2^{\text {nd }}$ year students, respectively. Most patients were students in LawSchool (16.25\%) and LiberalArtsSchool (12.50\%). Six severe cases were admitted at ChiangRaiRegionalHospital, PCR positive with $\mathrm{H} 1 \mathrm{~N} 1$, and administered with Tamiflu. Attack rate was 89.7/1000 population.

\section{Discussion}

Influenza A (H1N1) were rapidly transmitted in air conditioned classrooms. Urgent investigation and effective control measures could stop an outbreak within 10 days with no death reported.

Published: 11 May 2010

doi:10.1186/1742-4690-7-S1-P187

Cite this article as: Apidechkul: An outbreak of Influenza A (H1N1) 2009 at Mae Fah Luang University, Chiang Rai Province, northern Thailand.

Retrovirology 2010 7(Suppl 1):P187. 